\title{
„Wer klagt, ist verbrannt“
}

\section{Aus der Praxis von Berufungsverfahren}

\begin{abstract}
Professuren werden nicht immer so fair und transparent besetzt, wie die Verfahren es vorsehen. Mit den nachfolgenden Fallbeispielen aus der Berufungspraxis möchten die (anonymen) Autorinnen und Autoren einen Anstoß dazu geben, das Berufungsverfahren an den deutschen Universitäten neu zu überdenken. Es handelt sich um reale Vorgänge aus Berufungsverfahren für Professuren an deutschen Universitäten im Bereich der Sozial- und Rechtswissenschaften. Es geht den Autorinnen und Autoren nicht darum, auf ihr persönliches „Schicksal“ in Berufungsverfahren aufmerksam zu machen, sondern typische Vorgänge und grundsätzliche Probleme anzusprechen. Sie nehmen an, dass den Leserinnen und Lesern manches daran bekannt vorkommen wird. Um Rückschlüsse auf konkrete Verfahren zu erschweren, ziehen sie es vor, anonym zu bleiben. Zur Wahrung der Anonymität wurden einzelne Details leicht verändert.
\end{abstract}

Fall 1: Bester Freund und Bluthund

Bewerberin. Ich hatte mich um eine $\mathrm{W}_{3}$-Professur beworben und rechnete mir ganz gute Chancen aus; das Profil der Stelle passte perfekt auf mich. Eine meiner Mitbewerberinnen hatte bislang wenig publiziert und noch nicht einmal ihre Habilitationsschrift abgeschlossen; sie war aber mit dem einzigen engeren Fachkollegen F innerhalb des Fachbereichs gut befreundet. Als ich meinen - inhaltlich wenig kontroversen - Probevortrag hielt, schüttelte $F$ von Anfang an immer wieder energisch den Kopf. Er meldete sich in der Diskussion auch gleich zu Wort und unterstellte mir grobe Fehler an elementaren und völlig unproblematischen Stellen. Alles, was er angriff, war absoluter Konsens in unserem Fachgebiet - und ich hatte keine Zweifel daran, dass er das auch wusste. Aber niemand anderes in der Kommission war vom Fach und so stand es zwischen uns gewissermaßen „Aussage gegen Aussage". Ich versuchte, in ruhigem und freundlichen Ton zu erwidern, er hingegen wurde immer aggressiver, die Stimmung war unerträglich. Noch am selben Abend erhielt ich einen Anruf des Dekans. Er teilte mir mit, dass ich auf den 2. Platz der Liste gesetzt worden sei - direkt hinter der guten Freundin von F. Ich konnte meine Irritation nicht ganz verbergen. Der Dekan sagte: „Ja, also wir hatten Sie eigentlich auch alle auf Platz 1. Aber der Fachkollege F war da vehement dagegen. Sie haben ja selbst erlebt, dass die Chemie zwischen Ihnen nicht stimmt. Und er muss schließlich mit Ihnen zusammenarbeiten."

Fall 2: „Komplizierte“ Personen

Mitglied einer Berufungskommission. In einem Berufungsverfahren gab es einen fachlich hervorragend qualifizierten Bewerber, von dem allerdings kolportiert wurde, dass er im Umgang kompliziert und streitbar sei. Beim gemeinsamen Mittagessen der Kommission sagte ein Kollege mit Bezug auf den Bewerber: „Wenn ich Tennis spielen möch- te, dann suche ich mir ja nicht unbedingt jemanden aus, der mir die Bälle um die Ohren haut und auch noch über jeden Punkt diskutiert. Sondern jemanden, mit dem man in Ruhe ein paar Bälle spielen und danach noch gemütlich ein Bier trinken gehen kann." Der Bewerber wurde letztlich wegen vermeintlich fehlender fachlicher Passgenauigkeit für die Stelle nicht zu einem Vortrag eingeladen.

\section{Fall 3: Mittelmaß beruft Mittelmaß}

Bewerber. Ich bewarb mich von meiner Professorenstelle aus auf eine Professur an einer kleinen Universität in der Nähe meines Wohnorts. Außer mir hatten sich fast nur Privatdozenten beworben. Nachdem mein Vortrag gut gelaufen war und die Berufungskommission beraten hatte, unterhielt ich mich mit einem Kollegen, der mit dem dortigen Fachbereich gut vernetzt war. Er sagte, dass ich keine Chance auf die Stelle hätte. „An dem Fachbereich sind die Kollegen nicht besonders engagiert ... Mittelmaß beruft Mittelmaß. Die werden sich nie jemanden holen, der sie so sehr in den Schatten stellt." Tatsächlich wurde ich nicht auf die Liste gesetzt. Als Begründung wurde angegeben, dass man sich für eine neue - in der Ausschreibung nicht genannte - Ausrichtung der Stelle entschieden habe und ich in dieses Profil nicht passe. Ich überlegte kurz, ob ich gegen das Verfahren klagen sollte. Aber: Wer klagt, ist verbrannt. Also ließ ich die Sache auf sich beruhen.

\section{Fall 4: „Bitte nicht stören“}

Auswärtiger Gutachter. Nach meinem Eindruck aus mehreren Berufungsverfahren, an denen ich als auswärtiger Gutachter mitgewirkt habe, sind Berufungskommissionen vor allem daran interessiert, dass die von ihnen vorgeschlagene Liste nicht in Frage gestellt wird. Da es inzwischen 
hochschulrechtlich überwiegend untersagt ist, dem Gutachter die „Wunschliste“ mitzuteilen, habe ich verschiedene Wege kennengelernt, auf denen dennoch sichergestellt werden kann, dass der Gutachter nicht „,irrtümlich“ eine falsche Reihung vorschlägt:

a. Dem Gutachter werden schriftlich die Namen der zu begutachtenden Bewerberinnen und Bewerber übermittelt, allerdings durchaus nicht in alphabetischer Reihenfolge.

b. Der Gutachter erhält eine alphabetisch gereihte Liste, verbunden mit dem nicht $\mathrm{zu}$ übersehenden Hinweis des Kommissionsvorsitzenden: „Wenn Sie Rückfragen haben, können Sie mich gerne jederzeit anrufen."

c. Bevor die Kommission die Gutachter auswählt, ruft der Vorsitzende den ins Auge gefassten Kollegen an, fragt höflich nach der Bereitschaft, ein Gutachten zu erstellen, und erwähnt beiläufig die von der Kommission favorisierten Kandidaten. „Also, ich weiß nicht ... Sie sind natürlich völlig frei, Herr Kollege ... aber könnten Sie sich damit eventuell identifizieren?" Erst nach vorsichtiger Bejahung dieser Frage erhält man die offizielle Bitte, ein Gutachten zu erstellen.

\section{Fall 5: Die „Sonstigen“}

Mitglied einer Berufungskommission. Es geht um die Neubesetzung einer Professur im Forschungsbereich X. Herr A, der Sprecher des Forschungsbereichs, ruft mich an. „Wir brauchen für die Berufungskommission noch einen Vertreter des Mittelbaus. Wie wäre es mit Ihrer Mitarbeiterin M, auf die ist doch Verlass, nicht? Außerdem kann meine Verwaltungsangestellte für das sonstige Personal mitwirken. Und als weiteres Mitglied aus unserer Fakultät sollten wir den Kollegen $\mathrm{K}$ fragen, der kommt im Zweifel sowieso nicht zu den Sitzungen, weil er keine Zeit hat. Als auswärtiges Mitglied können wir die Kollegin F einladen, die versteht von unserem Forschungsbereich nicht viel, wird also keinen Ärger machen." So wird dann auch verfahren.

Nachdem die Kommission über die gewünschte Liste abgestimmt hat, geht es um die Benennung zweier auswärtiger Gutachter. Auch hier hat Herr A schon konkrete Vorstellungen: „Am besten frage ich den Kollegen L, der ist mir noch einen Gefallen schuldig, weil ich für seine Fakultät begutachtet habe. Und dann nehmen wir vielleicht noch Frau N; von der weiß ich, dass sie mit dem Ersten auf unserer Liste befreundet ist. Da kann nicht viel schiefgehen." Es geht auch nichts schief: Die Liste wird so beschlossen, wie Herr A es vorher mit seinen engeren Fachkollegen besprochen hatte.

Fall 6: Vorsicht, Frauen! (1)

Mitglied einer Berufungskommission. Während meiner Habilitationszeit war ich Vertreterin des Mittelbaus in einer Berufungskommission. Die Stelle sollte nach Meinung der beteiligten Kommissionsmitglieder des Fachbereichs ein Mann erhalten, der den Lehrstuhl während der Vakanz vertreten hatte. Eingegangene Bewerbungen weiblicher Kandidaten wurden seitens des Dekans mit dem Kommentar versehen, man müsse Begründungen finden, weshalb die „Frauen gar nicht erst eingeladen werden. Dann macht die Gleichstellungsbeauftragte auch keinen Ärger.“ So geschah es dann auch. In einem anderen Berufungsverfahren mit ähnlicher Konstellation lernte ich, dass auf keinen Fall gegen das „eherne Gesetz" verstoßen werden darf, wonach ein älterer, männlicher Professor stets einer jüngeren Privatdozentin vorzuziehen ist. So etwas könne man dem männlichen Kollegen „nicht antun“.

Fall 7: Vorsicht, Frauen! (2)

Mitglied einer Berufungskommission. Das Rektorat meiner Universität hatte darum gebeten, in unserer Fakultät wegen des geringen Frauenanteils bei Ausschreibungen Frauen, die für die ausgeschriebene Stelle geeignet sind, aktiv zur Bewerbung aufzufordern. Diese Vorgabe wurde in der Berufungskommission, an der ich teilnahm, durchaus kreativ ausgelegt. Nachdem die Kommission sich zugunsten eines älteren Herrn gegen eine jüngere Bewerberin ausgesprochen hatte, gab es Ärger mit der Gleichstellungsbeauftragten. Daraufhin rief der Kommissionsvorsitzende die weibliche Bewerberin persönlich an - und bat sie, ihre Bewerbung doch bitte zurückzuziehen. Sie tat dies dann auch, und zur (fast) allgemeinen Erleichterung durfte der männliche Wunschkandidat in der Universität Einzug halten.

Fall 8: Rache ist süß

Bewerberin. Ich bewarb mich von einer Professur aus auf eine Ausschreibung der Universität X, deren Anforderungsprofil auf mich passte. Maßgebliche Mitglieder der Berufungskommission waren vier engere Fachkollegen, darunter Professor A, der kurze Zeit zuvor auf einer Berufungsliste hinter mir gelandet war. $\mathrm{Zu}$ einem Bewerbungsgespräch wurde ich nicht eingeladen. Auf meine Nachfrage teilte mir der Kommissionsvorsitzende mit, dass im Vorfeld der Einladungen externe Gutachter damit beauftragt worden seien, eine Stellungnahme dazu abzugeben, ob ich eingeladen werden müsse. (Am Rande bemerkt: ein in unserem Fach völlig ungewöhnlicher Schritt!) Der Dekan leitete mir Auszüge aus dem externen Gutachten zu, die einer fachlichen Vernichtung gleichkamen. Einem Leser des Gutachtens musste es unverständlich sein, wie es zu meiner Habilitation durch einen Fachbereich einer deutschen Universität hatte kommen können - geschweige denn zu zwei Rufen an andere Universitäten. Und der Dekan legte noch nach: Sollte ich das Verfahren weiter verfolgen, könne nicht sicher vermieden werden, dass die Angelegenheit Wellen schlägt. Klar, die Nestbeschmut- 
zerin würde zumindest community-öffentlich an den Pranger gestellt werden.

Fall 9: „Wir sind uns doch alle einig“

Bewerber. Als in einigen theoretischen Fragen vom „Mainstream" in meinem Fach abweichender Wissenschaftler habe ich in den vergangenen Jahren häufig folgende Erfahrung gemacht: Andersdenken ist für viele kein wissenschaftliches Gütesiegel, sondern wird als Bedrohung empfunden. Diese Haltung wird für den Andersdenkenden dann zum Problem, wenn er sich in die strukturell unterlegene Position des Bewerbers in einem Berufungsverfahren begibt. Kommt es zur Einladung zu einem Probevortrag, so gleicht diese Zusammenkunft nicht selten einem Tribunal, in dem allein die Verteilung der bestehenden Machtverhältnisse dazu führt, dass der Bewerber entweder als gescholtener Schuljunge oder - im Fall des Zurwehrsetzens - als Querulant und insgesamt unangenehmer Zeitgenosse vom Platz geht. Zu gewinnen gibt es da nichts.

Als Ausdruck meiner Unzulänglichkeit wurde mir regelmäßig entgegengehalten, dass mir die „Anschlussfähigkeit" fehle (verstanden als Anpassungsfähigkeit an die von den hiesigen Kollegen vertretenen Auffassungen). Und: Wenn auch sonst die Studierenden und ihre Bedürfnisse in Berufungsverfahren zumeist eine allenfalls nachgeordnete Rolle spielen, dienten sie nun als Pfeilspitze der Kritik: Meine „kruden“ Thesen dürfen erst gar nicht an die Ohren der Jugend herangetragen werden. Sie könnte am Ende verwirrt werden - da belassen wir doch lieber alles beim Alten, nicht wahr?

\section{Aufruf zur Benennung von Kandidatinnen und Kandidaten für die Präsidiumswahlen 2021}

Im Auftrag der Präsidentin der DMV teile ich Ihnen mit, dass zum 1.1.2022 drei Positionen im DMV-Präsidium neu zu besetzen sind. Alle DMV-Mitglieder können Wahlvorschläge machen. Diese müssen nach Paragraph $2 \mathrm{~b}$ der Wahlordnung von mindestens zehn Mitgliedern unterstützt werden und bis zum 6. Mai 2021 bei der Geschäftsstelle eingegangen sein.

Weiterhin ist nach Paragraph 2a der Wahlordnung das Präsidium verpflichtet, für jede frei werdende Posi- tion im Präsidium wenigstens einen Kandidaten/eine Kandidatin zu benennen.

Ich möchte alle Mitglieder nachdrücklich bitten, Vorschläge zu machen. Es wäre wünschenswert, mehr Kandidaten als Positionen zur Auswahl zu haben.

Der Schriftführer

Daniel Grieser 